\title{
Seroprevalence of Borrelia burgdorferi sensu lato and Anaplasma phagocytophilum in Danish horses
}

\author{
Marie GB Hansen ${ }^{1,2}$, Mette Christoffersen², Line R Thuesen', Morten R Petersen², Anders M Bojesen ${ }^{\text {* }}$
}

\begin{abstract}
Background: Borrelia burgdorferi sensu lato and Anaplasma phagocytophilum are able to infect horses. However, the extend to which Danish horses are infected and seroconvert due to these two bacteria is unknown. The aim of the present study was to evaluate the seroprevalence of B. burgdorferi sensu lato and A. phagocytophilum in Danish horses.

Methods: A total of 390 blood samples collected from all major regions of Denmark and with a geographical distribution corresponding to the density of the Danish horse population were analyzed. All samples were examined for the presence of antibodies against B. burgdorferi sensu lato and $A$. phagocytophilum by the use of the SNAP® 4 DX ${ }^{\oplus}$ ELISA test.
\end{abstract}

Results: Overall, $29.0 \%$ of the horses were seropositive for B. burgdorferi sensu lato whereas $22.3 \%$ were seropositive for A. phagocytophilum.

Conclusions: Antibodies against B burgdorferi sensu lato and A. phagocytophilum are commonly found among Danish horses thus showing that Danish horses are frequently infected by these organisms.

\section{Background}

The vector-borne bacteria Borrelia burgdorferi sensu lato (B. burgdorferi s. 1.) and Anaplasma phagocytophilum infect horses in those parts of the world where Ixodes spp. are present. It has been estimated that up to $30-40 \%$ of horses in an endemic area are seropositive for B. burgdorferi s. 1. [1]. Most of these horses will remain asymptomatic, while $5-10 \%$ of them are likely of developing clinical signs [1-4]. Similar, a previous study on A. phagocytophilum, have indicated that up to $50 \%$ of seropositive horses in endemic areas undergo a subclinical infection [5]. It is assumed that clinical equine granulocytic anaplasmosis is an overseen condition in most of Europe, as most horses recover spontaneously and therefore do not attract the attention of clinicians [6]. In Denmark, B. burgdorferi s. l. and A. phagocytophilum are transferred by the tick Ixodes ricinus. A Danish study from 2005 revealed that the tick density varies

\footnotetext{
* Correspondence: miki@life.ku.dk

'Department of Veterinary Disease Biology, Faculty of Life Sciences, University of Copenhagen, Stigbøjlen 4, DK-1870 Frederiksberg C, Denmark
}

substantially between different regions of Denmark with highest density on the island of Bornholm (0.5-1.0 ticks/ min. flagging) followed by Zealand, Funen, Middle-Jutland and East-Jutland (0.25-0.5 ticks/min. flagging), South-Jutland (0.15-0.25 ticks/min. flagging), and West and North-Jutland (0-0.15 ticks/min. flagging) [7]. An apparent increase in the tick density from 1984 to 1998 [8] fits well with a positively correlated relationship between warmer winters and longer spring and autumn periods and the density of $I$. ricinus $[9,10]$.

The seroprevalence of B. burgdorferi s. l. and A. phagocytophilum among horses in Denmark has to our knowledge never been evaluated. Recent European studies on the seroprevalence of B. burgdorferi $\mathrm{s}$. l. shows a prevalence of $47.8 \%$ seropositive horses in Slovakia [11], $25.6 \%$ in Poland [12], $16.8 \%$ in Sweden [13], 16.1\% in Germany [14] and 6.3\% in Turkey [15]. The seroprevalence of A. phagocytophilum in Europe varies from $83.3 \%$ in Holland [6], $16.7 \%$ in Sweden [13], $11.3 \%$ in France [16], 8.1\% in Italy [17,18] to $6.5 \%$ in Spain [19]. Furthermore, a Swedish study reported that $4.5 \%$ of the examined horses were seropositive for both $B$.
C Biomed Central 
burgdorferi s. 1. and A. phagocytophilum [13]. In 2005, Danish researchers made a seroprevalence study on the distribution of B. burgdorferi s. 1 . and A. phagocytophilum antibodies in Danish deer. The overall seroprevalence was $36.6 \%$ for $B$. burgdorferi s. 1 . Significant regional differences were found when Jutland was compared with the islands (Funen, Zealand, Lolland, Falster and Bornholm) with a seroprevalence of $27.1 \%$ versus $46.7 \%$, respectively. In the case of $A$. phagocytophilum, all tested deer in three districts of North-Jutland were antibody negative although the average seroprevalence was found to be $42.6 \%$ ranging from $39.6 \%$ in Jutland to $47.6 \%$ of the islands [7].

The aim of the present study was to evaluate the seroprevalence of B. burgdorferi s. 1. and A. phagocytophilum in Danish horses.

\section{Materials and methods Sample size}

The sample size was calculated using the following formulas:

$$
\begin{aligned}
& n=N x /\left((\mathrm{N}-1) E^{2}+x\right) \\
& E=\operatorname{Sqrt}[((N-n) x / n(N-1)] \\
& x=Z(c / 100)^{2} r(100-r)
\end{aligned}
$$

where $n$ is the sample size, $E$ is the margin of error, $r$ is the fraction of response that we are interested in and $Z(c / 100)$ is the critical value for the confidence level of $c$.

As the prevalence of horses seropositive for B. burgdorferi s. 1 . and A. phagocytophilum vary considerably in areas comparable to Denmark [11-14], calculations were based on a situation where the test results from each individual horse could have 50\%-50\% test outcome ( $r=$ $50 \%)$. A difference of more than $5 \%(E)$ to the expected $50 \%-50 \%$ outcome with a confidence of $95 \%$ (c) should be detected. Thus, with an estimated population of 200,000 horses $(N)$ in Denmark, a sample size including 384 individuals was proposed. As the test kits came as pre-packed batches, 390 individual blood samples were taken.

\section{Selection of horses}

No formal randomized selection of horses was applied but an even representation of samples from all regions in Denmark was aimed at. Denmark is officially divided into five administrative regions including the Capital Region (C), Region Zealand (Z), Region South-Denmark (SD), Region Central-Jutland (CJ) and Region North-Jutland (NJ) (Figure 1). Assuming that the horse population is evenly distributed between the five regions, a sample size of 78 horses was used for each region. Within each region, horses were selected based on availability due to previous contact to individual horse owners, horse studs and clinical veterinary practices. Approximately one third of the samples were obtained from horses admitted to four equine veterinary clinics. The remaining samples were taken during visits to various size breeding farms, horse riding schools and farms. No horses with signs or a recent history of untreated infectious diseases were included.

\section{Serological evaluation of blood samples}

A total of 390 blood samples were collected in EDTA blood collection tubes. All blood samples were analyzed immediately by a SNAP ${ }^{\oplus} 4 \mathrm{DX} \bullet$ ELISA test (IDEXX Laboratories, Taastrup, Denmark) according to the manufactures instructions.

\section{Putative risk factors}

Every horse owner was asked to provide information with regard to putative risk factors including the breed, gender, age, use and housing, respectively. For each factor, 25 categories were established; "no answer" was included as a category. Gender included mare, stallion and gelding. Age was classified into groups of 1-4 years, 5-10 years, 11-20 years, and over 21 years. Housing facilities included pasture and pasture in combination with stabled housing, and access to pasture in the summer period was categorized as 1-3 months and $>3$ months. The horses were used for breeding, competitions/races, riding school and for leisure riding. Another risk factor was the use of the horse; it included the categories not used for riding, primarily used for leisure riding in the terrain or primarily used on a riding ground. Finally it was noted if the horse has been used for breeding.

\section{Statistical analyses}

The ELISA results and their association with the putative risk factors were statistically analyzed using the PROC logistic procedure in SAS 9.1 (SAS Institute, Cary, NC, USA). A logit-transformation was used to estimate the relation between the outcome and the explanatory variables.

The model was given by:

Logit $\left(p_{i}\right)=\alpha+b\left(\right.$ bacteria $\left._{i}\right)+c\left(\right.$ region $\left._{i}\right)+d\left(\right.$ gender $\left._{i}\right)+$

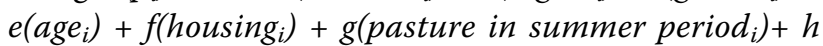
$\left(\right.$ breed $\left._{i}\right)+i\left(\right.$ use $\left._{i}\right)+j\left(\right.$ riding $\left._{\text {type }}\right)+k\left(\right.$ breeding $\left._{i}\right)$

Where $P_{i}$ is the dependent variable thefollowing outcomes was possible i) B. burgdorferi s. 1. ii) A. phagocytophilum, iii) B. burgdorferi s. l. or A. phagocytophilum iv) both $B$. burgdorferi s. 1 . and A. phagocytophilum), $\alpha$ is the intercept, and ${ }_{i}$ refers to the level of categories to the respective risk factor. The PROC logistic procedure tested two-way interactions between the different explanatory variables (risk factors). From the full model including all explanatory variables, a backward elimination was used to exclude non-significant variables. The parametric statistical Wald test was used to exclude the most non-significant variables until all variables in the model were significant. 


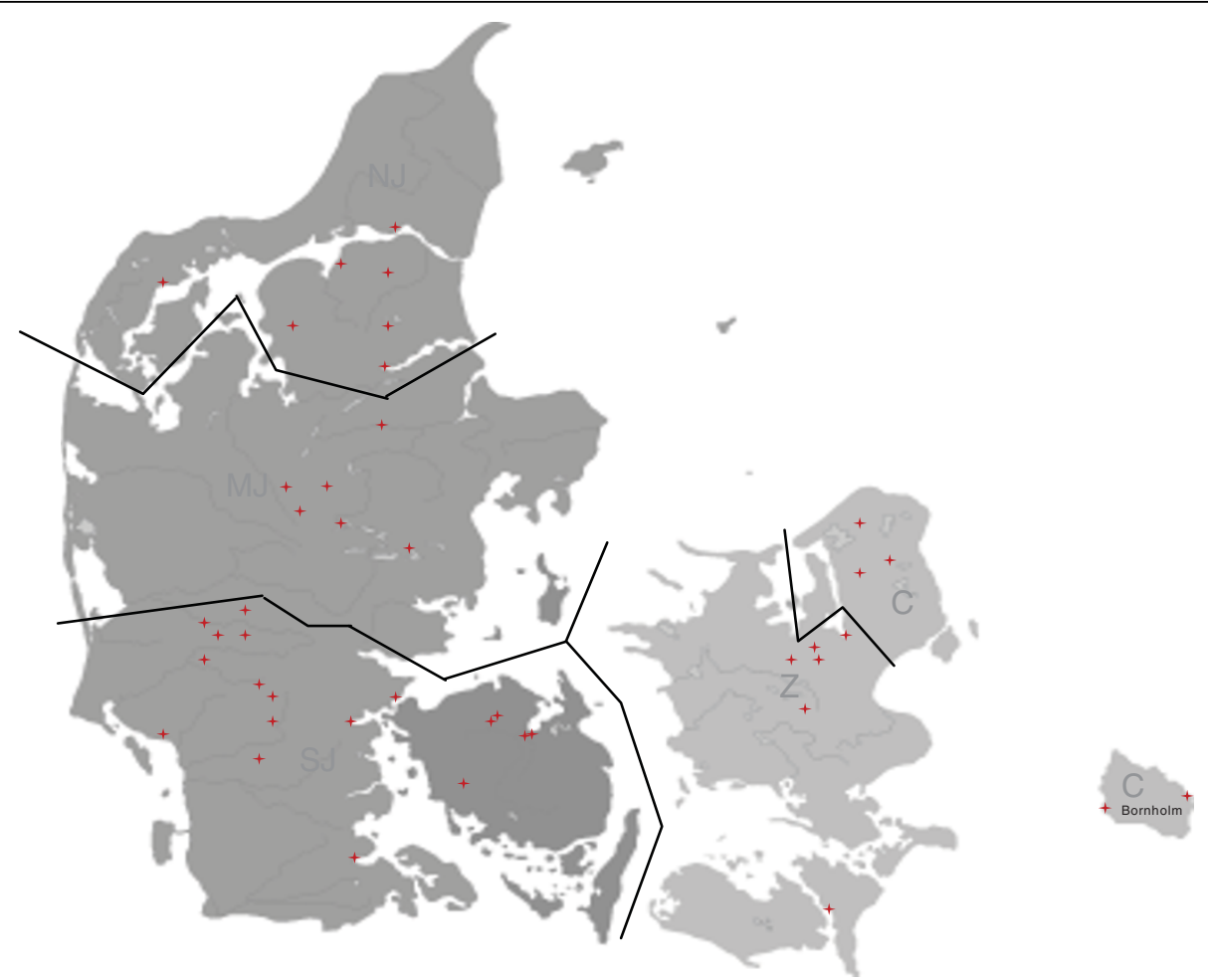

Figure 1 Geographical location of sample sites (stars) within each of the five administrative regions of Denmark. (NJ) Northern Jutland, (MJ) Mid Jutland, (SJ) Southern Jutland, (Z) Zeeland and (C) Capital region.

To test the difference between the seroprevalence of B. burgdorferi s. l., A. phagocytophilum and the different regions, Chi square test or Fischer's exact test were used.

All statistical calculations were made with the software SAS 9.1. The level of significance was set to $\mathrm{P}<0.05$.

\section{Results}

\section{Sampling}

The sampled horses consisted of 222 mares, 135 geldings and 33 stallions. The breeds included Icelandic horse (182), Danish Warmblood (67), Jysk horse (11), Standardbred (11), Shetland Pony (10), Oldenburg (6), Friesian (6), Trakehner (5), Hanoverian (5), North Bagge (5), Connemara (4), Arabs (4) and other/mixed breeds (74). Horses aged 1 to 30 years (Mean $=9.2$ years, SD \pm 6.0) were included. All samples were obtained in the period from the $5^{\text {th }}$ of April to the $11^{\text {th }}$ of May 2009. The horses sampled originated from herds based in 42 individual postal codes (Figure 1). Of the horses tested through the participating veterinary clinics, 6-8 were under treatment and in the late recovery phase of an antibiotic treatment regimen, whereas the remaining horses were prophylactic cases including vaccination, teeth management, and the vast majority were subjected to assisted reproduction.

\section{Serology}

The over-all seroprevalence of B. burgdorferi s. l. was $29.0 \%$ and $22.3 \%$ for $A$. phagocytophilum. The highest seroprevalence of $33.3 \%$ for B. burgdorferi s. l. was found in the Region of South-Denmark while only $24.4 \%$ of the samples from Region of North-Jutland were positive. The highest $A$. phagocytophilum seroprevalence (33.3\%) was found in the Region of Zealand while the lowest $(16.7 \%)$ was found in the Capital Region. Despite the seemingly high regional differences in seroprevalence for either organism, this could not be demonstrated statistically $(P \geq 0.29)$. The regional seroprevalence distribution is summarized in Table 1 .

Table 1 Seroprevalence of Borrelia burgdorferi sensu lato and Anaplasma phagocytophilum in 390 Danish horses.

\begin{tabular}{lcc}
\hline Region & Borrelia-positive (\%) & $\begin{array}{c}\text { Anaplasma-positive } \\
\text { (\%) }\end{array}$ \\
\hline Region North-Jutland & $19(24.4)$ & $18(23.1)$ \\
Region Central-Jutland & $21(26.9)$ & $16(20.5)$ \\
Region South-Denmark & $26(33.3)$ & $14(18.0)$ \\
Region Zealand & $25(32.1)$ & $26(33.3)$ \\
Capital Region & $22(28.2)$ & $13(16.7)$ \\
Denmark (Total) & $113(29.0)$ & $87(22.3)$ \\
\hline
\end{tabular}

A total of 78 horses were sampled from each region. 
There was a considerable with-in region variation of the seroprevalences e.g. the Capital Region, which had an overall low seroprevalence for both $B$. burgdorferi s. l. and $A$. phagocytophilum. However, the seroprevalence for the island Bornholm, which belongs to the Capital Region was $60 \%$ for B. burgdorferi s. 1 . and $50 \%$ for $A$. phagocytophilum. Omitting the results from Bornholm, the Capital Region had a seroprevalence of $17.2 \%$ for $B$. burgdorferi s. 1 . and $5.2 \%$ for A. phagocytophilum. There was a significant lower seroprevalence of both B. burgdorferi s. $1 .(P=0.017)$ and Anaplasma phagocytophilum $(P<0.001)$ in the Capital Region (Bornholm excluded) compared to Bornholm.

No significant association was found between any of the proposed risk factors and the occurrence of antibodies against $B$. burgdorferi s. l. (outcome (i). On the contrary, a significant correlation $(P \leq 0.03)$ was found between the age of the horses and the presence of $A$. phagocytophilum antibodies (outcome ii). Horses aged 11 to 20 years had an odds ratio (OR) of 2.3 (with a 95\% confidence interval of 1.2-4.6) for being seropositive for $A$. phagocytophilum whereas horses $\geq 21$ years had an OR of 3.3 (with a 95\% confidence interval 1.1-10.5) for being seropositive compared with horses aged 1-4 years, respectively. There was no significant association between other risk factors and presence of $A$. phagocytophilum antibodies. Eleven percent of the examined horses were seropositive for both B. burgdorferi s. l. and A. phagocytophilum. It appeared that the OR for horses seropositive for B. burgdorferi s. 1. was 3.1 (with a 95\% confidence interval of 1.9-5.0) when simultaneously being seropositive for A. phagocytophilum (outcome iii). Likewise, the OR for horses seropositive for $A$. phagocytophilum was 3.3 (with a confidence interval of 1.9-5.4) for simultaneously being seropositive for B. burgdorferi s. 1. (outcome iii) Thus, there was a significant association between the occurrence of antibodies against $B$. burgdorferi s. 1. and $A$. phagocytophilum $(P \leq 0.0001)$ (outcome iiii) There was no significant association between the other factors investigated and the occurrence of a simultaneous infection with $B$. burgdorferi s. 1. and A. phagocytophilum.

\section{Discussion}

Comparing the seroprevalences of B. burgdorferi s. l. and A. phagocytophilum with results from neighboring European countries [insert refs], the seroprevalences found in this study are considerably higher. One important point explaining this difference could relate to sampling bias i.e. horses included in our study could have had a higher exposure risk to infected ticks than the general population. Although a formal randomized selection of horses was not applied, the considerable differences in geographic location, breed, age and use of the horses sampled is believed to provide a good approximation of the general horse population in Denmark. Furthermore, none of the horses included this study were selected because they exhibited or have had clinical signs of equine borreliosis or equine granulocytic anaplasmosis. On the contrary, previous studies have shown a steady increase in the ticks density, which has been attributed to climatic changes [8] and an increased infection rate among the ticks [21], which may prove to be more likely reasons for the higher prevalences observed. Whether the differences in the prevalences in Denmark versus Sweden and Germany $[13,14]$ are due to an increase in the number of infected ticks since the latter investigations were performed, or whether the prevalence in Denmark in fact is higher will however remain unknown until data from prevalence studies with a comparable design and method from all countries in the region are performed. Differences in study design e.g. serological test method and statistical approach for the analysis of the results currently makes comparison of previous results difficult.

The highest density of ticks has previously been found on Bornholm, whereas a lower density was observed in the major Danish islands and in Jutland [7]. In addition to this, a Danish study of the seroprevalence of B. burgdorferi s. 1. in deer showed that the risk of becoming infested with a tick positive for B. burgdorferi s. 1. was greatest in the Capital Region followed by the Region of Zealand and the regions of Jutland, respectively. Interestingly, the risk of a deer being positive for B. burgdorferi s. 1. antibodies was two times higher for Bornholm (part of the Capital Region) than for the island Zealand in general [22]. The present study shows that most of the horses seropositive for B. burgdorferi s. l. were found in the Region of South-Denmark followed by the Region of Zealand, the Capital Region, and Region of Central-Jutland and the Region of North-Jutland, thereby largely reflecting the tick density. In cases where the results did not match the deer study [ref], this may reflect differences as to the locality sampled within the regions compared. In accordance with the above, the study also shows that the prevalence of horses seropositive for B. burgdorferi s. l. on the island of Bornholm was two times higher than for Zealand, when the results for Bornholm was omitted in the overall result of the Capital Region and the Region of Zealand. We analyzed blood samples taken during spring, which likely influenced the number of seropositive individuals negatively as the likelihood of becoming seropositive later during the summer and autumn due to a longer exposure and risk of being infested by an infected tick [13].

The SNAP ${ }^{\oplus} \mathrm{DX}{ }^{\bullet}$ test was developed for screening of Dirofilaria immitis antigen and antibodies to $A$. phagocytophilum, B. burgdorferi and Ehrlichia canis in canine 
serum, plasma or whole blood [23,24]. However, $\mathrm{SNAP}^{\star} 4 \mathrm{DX}{ }^{\bullet}$ has previously been evaluated and found useful to detect antibodies against B. burgdorferi s. 1 . and $A$. phagocytophilum in equine samples [23]. Compared to Western immunoblot, the $\mathrm{SNAP}^{\bullet} 4 \mathrm{DX}{ }^{\bullet}$ was found to have a sensitivity of $100 \%$ and a specificity of 95\% for detection of antibodies against B. burgdorferi s. 1. in equine samples. Detecting $A$. phagocytophilum antibodies in equines by the $\mathrm{SNAP}^{\oplus} 4 \mathrm{DX}{ }^{\bullet}$ test comparison to the indirect immunofluorescence assay showed a sensitivity and a specificity of $100 \%$ [25]. Johnson et al. [ref] found the $\mathrm{SNAP}^{\star} 4 \mathrm{DX}{ }^{\bullet}$ test less sensitive (63\%) yet $100 \%$ specific when comparing it to Western blotting and a C6-ELISA during a longer course of experimental infection [28]. By using ELISA in the form of a SNAP ${ }^{\oplus} \mathrm{DX}^{\odot}$ test it should be noted that this method detects both active and previous infections with measurable antibody levels [20]. From previous studies, the antibody levels in horses have been found detectable for up to 2 years $[20,26]$. In these, the clinically affected horses had the highest ELISA titers compared to horses with subclinical infections [1]. From studies with horses experimentally infected with $B$. burgdorferi s. 1 . and subsequently analyzed by ELISA, it appeared that even in cases where the horses had no clinical signs, antibodies were detected within 5-6 weeks. Antibody levels rose to a maximum over the following 3-4 months after which they remained static for at least 9 months [27]. However, another study has shown that individual horses, despite being positive by PCR and cell culture, did not remain seropositive by the $\mathrm{SNAP}^{\oplus} 4 \mathrm{DX}^{\oplus}$ test over a period of 9 months [28]. The above indicates that the antibody level may be detectable by some ELISA tests at least 9 months post-infection with B. burgdorferi s. 1 . but presumably for a shorter period using the $\mathrm{SNAP}^{\oplus} 4 \mathrm{DX}^{\oplus}$ test. Experimental infections in horses inoculated intravenously with $A$. phagocytophilum resulted in seroconversion 6-8 days post inoculation and achieved a maximum ELISA titer at day 10-30. The antibodies remained detectable for up to 5 months [28]. It is well established that horses acquiring a natural infection with A. phagocytophilum mount a slower antibody response, likely since the burden of infection typically is lower and delivered over a prolonged period [29]. Despite a longer incubation period, prior seroconversion from a natural infection versus the intravenously administered infections, the antibody levels were similar after 30 days [30]. Therefore, it is reasonable to assume that an ELISA may have a positive outcome for up to 5 months post-infection with $A$. phagocytophilum. The results found in this study therefore likely reflect the proportion of horses that have been infected with $B$. burgdorferi s. 1. within the last 9 months or $A$. phagocytophilum within the last 5 months from the time of sampling and not only horses with an active infection at the time of blood sampling.

\section{Conclusions}

The present study demonstrates that antibodies against $B$. burgdorferi s. 1. and A. phagocytophilum can be commonly found in Danish horses. The findings warrant further attention to these infections in horses particularly with regard to improved means for detection of active infections, which may contribute to a better general understanding of these diseases and their impact on horse behavior and welfare.

\section{Acknowledgements}

Idexx Laboratories and Kruuse A/S are thanked for providing discounted ELISA kits.

\section{Author details}

${ }^{1}$ Department of Veterinary Disease Biology, Faculty of Life Sciences, University of Copenhagen, Stigbøjlen 4, DK-1870 Frederiksberg C, Denmark. ${ }^{2}$ Department of Large Animal Sciences, Faculty of Life Sciences, University of Copenhagen, Dyrlægevej 68, DK-1870 Frederiksberg C, Denmark.

\section{Authors' contributions}

MGBH participated in the design of the study and carried out the sampling and ELISA tests, contributed to the statistical analysis and drafted the manuscript. MC and LRT performed the statistical analysis. MRP participated in the design of the study and helped to draft the manuscript. AMB participated in the design of the study, coordinated the activities and helped to draft the manuscript. All authors read and approved the final manuscript.

\section{Competing interests}

The authors declare that they have no competing interests.

Received: 28 September 2009

Accepted: 18 January 2010 Published: 18 January 2010

\section{References}

1. Manion TB, Bushmich SL, Mittel L, Laurendeau M, Werner H, Reilly M: Lyme disease in horses: Serological and antigen testing differences. 44th Proceedings of the Annual Convention of the AAEP: 6-9 December 1998; Baltimore Manion TB, Bushmich SL 1998, 144-145.

2. Bushmich SL: Lyme borreliosis in domestic animals. J Spiro Tick Dis 1994, 1:24-28.

3. Magnarelli LA, Anderson JF, Shaw E, Post JE, Palka FC: Borreliosis in equids in northeastern United States. Am J Vet Res 1988, 49:359-362.

4. Myhre G, Orcutt R: Lyme disease: Insight into prevalence diagnosis and treatment. J Equi Vet Sci 2008, 28:390-391.

5. Madigan JE, Hietala S, DeRock E: Seroepidemiologic survey of antibodies to Ehrlichia equi in horses of northern California. J Am Vet Med Assoc 1990, 196:1962-1964.

6. Butler CM, Nijhof AM, Jongejan F, Kolk van der JH: Anaplasma phagocytophilum infection in horses in the Netherlands. Vet Rec 2008, 162:216-217

7. Skarphédinsson S, Jensen PM, Kristiansen K: Survey of tickborne infections in Denmark. Emerg Infect Dis 2005, 11:1055-1061.

8. Jensen PM, Frandsen F: Temporal risk assesment for Lyme borreliosis in Denmark. Scand J Infect Dis 2009, 32:539-544.

9. Bennet L, Halling A, Berglund J: Increased incidence of Lyme borreliosis in southern Sweden following mild winters and during warm humid summers. Euro J Clin Microbiol Infect Dis 2006, 25:426-432.

10. Lindgren $E$, Tälleklint $L$, Polfeldt $T$ : Impact of climatic change on the northern latitude limit and population density of the diseasetransmitting European tick Ixodes ricinus. Environ Health Perspect 2000, 108:119-123. 
11. Stefancíková A, Derdáková M, Škardová I, Szestáková E, Cisláková L, Kovácová D, Stanko M, Petko B: Prevalence of antibodies to Borrelia burgdorferi in horses of east Slovakia. Vet Med Czech 2000, 45:227-231, [in Czech].

12. Stefanciková A, Adaszek L, Petko B, Winiarczyk S, Dudinák V: Serological evidence of Borrelia burgdorferi sensu lato in horses and cattle from Poland and diagnostic problems of Lyme borreliosis. Ann Agric Environ Med 2008, 15:37-43.

13. Egenvall $A$, Franzén $P$, Gunnarsson $A$, Engvall EO, Vågsholm I, Wikström UB, Artursson K: Cross-sectional study of the seroprevalence to Borrelia burgdorferi sensu lato and granulocytic Ehrlichia spp. and demographic, clinical and tick-exposure factors in Swedish horses. Prev Vet Med 2001, 49:191-208,

14. Käsbohrer A, Schönberg A: Serologic studies of the occurrence of Borrelia burgdorferi in domestic animals in Berlin (West). Berl Münch Tierärztl Wochenschr 1990, 103:374-378, [in German].

15. Bhide M, Yilmaz Z, Golcu E, Torun S, Mikula I: Seroprevalence of antiBorrelia burgdorferi antibodies in dogs and horses in Turkey. Ann Agric Environ Med 2008, 15:85-90

16. Leblond A, Pradier S, Pitel PH, Fortier G, Boireau P, Chadoeuf J, Sabatier P. An epidemiological survey of equine anaplasmosis (Anaplasma phagocytophilum) in southern France. Rev Sci Tech 2005, 24:899-908, [in French].

17. Passamonti F, Fabrizia V, Katia C, Stefano C, Giacomo C, Luisa MM, Daniela PF, Andrea VS, Mauro C: Anaplasma phagocytophilum in horses and ticks: A preliminary survey of central Italy. Comp Immunol Microbiol Infect Dis 2010, 33:73-83.

18. Torina A, Vicente J, Alongi A, Scimeca S, Turlá R, Nicosia S, DiMarco V, Caracappa S, de la Fuente J: Observed prevalence of tick-borne pathogens in domestic animals in Sicily, Italy during 2003-2005. Zoonoses Public Health 2007, 54:8-15.

19. Amusategui I, Sainz A, Tesouro MA: Serological evaluation of Anaplasma phagocytophilum infection in livestock in northwestern Spain. Ann N Y Acad Sci 2006, 1078:487-490.

20. Dessau RB, Bangsborg JM, Ejlertsen T, Hansen K, Lebech A, Østergaard C: Laboratory diagnostics of infections caused by Borrelia burgdorferi. Ugeskr Læger 2006, 168:2805-2807, [in Danish].

21. Vennestrøm J, Egholm H, Jensen PM: Occurrence of multiple infections with different Borrelia burgdorferi genospecies in Danish Ixodes ricinus nymphs. Parasitol Int 2008, 57:32-37.

22. Jensen PM, Hansen H, Frandsen F: Spartial risk assesment for Lyme borreliosis in Denmark. Scand J Infect Dis 2000, 32:545-550.

23. IDEXX Laboratories: Package Insert. 2009http://www.idexx.com/ animalhealth/testkits/4dx/060505707.pdf.

24. IDEXX Laboratories: SNAP 4Dx Test. 2009http://www.idexx.com/ animalhealth/testkits/4dx/.

25. Chandrashekar R, Daniluk D, Moffitt S, Williams J: Serologic diagnosis of equine borreliosis: Evaluation of an in-clinic enzyme-linked immunosorbent assay (SNAP 4Dx). Intern J Appl Res Vet Med 2008, 6:145150.

26. Reed SM, Bayly WM, Sellon DC: Equine Internal Medicine Louis: Saunders, 1 2004.

27. Chang YF, Novosol V, McDonough SP, Chang C-F, Jacobson RH, Divers T, Quimby FW, Shin S, Lein DH: Experimental infection of ponies with Borrelia burgdorferi by exposure to Ixodid ticks. Vet Pathol 2000, 37:68-76.

28. Johnson AL, Divers TJ, Chang Y-F: Validation of an in-clinic enzyme-linked immunosorbent assay kit for diagnosis of Borrelia burgdorferi infection in horses. J Vet Diagn Invest 2008, 20:321-324.

29. Pusterla N, Lutz H, Braun U: Experimental infection of four horses with Ehrlichia phagocytophila. Vet Rec 1998, 143:303-305.

30. Franzén P, Aspan A, Egenvall A, Gunnarsson A, Åberg L, Pringle J: Acute clinical, hematologic, serologic and polymerase chain reaction findings in horses experimentally infected with a European strain of Anaplasma phagocytophilum. J Vet Intern Med 2005, 19:232-239.

doi:10.1186/1751-0147-52-3

Cite this article as: Hansen et al: Seroprevalence of Borrelia burgdorferi sensu lato and Anaplasma phagocytophilum in Danish horses. Acta Veterinaria Scandinavica 2010 52:3.

Publish with BioMed Central and every scientist can read your work free of charge

"BioMed Central will be the most significant development for disseminating the results of biomedical research in our lifetime. "

Sir Paul Nurse, Cancer Research UK

Your research papers will be:

- available free of charge to the entire biomedical community

- peer reviewed and published immediately upon acceptance

- cited in PubMed and archived on PubMed Central

- yours - you keep the copyright 